\title{
A rubber tube in the bladder as a complication of autoerotic stimulation of the urethra
}

\author{
Konstantinos Stamatiou, Hippocrates Moschouris
}

Tzaneio Hospital, Pireas, Greece.

\begin{abstract}
Summary Self-insertion of foreign bodies in the urethra is most commonly associated with sexual or erotic arousal of adolescents with mental health disorders. Rarely it may practiced by healthy adults for masturbation. Migration of foreign bodies used for the abovementioned purpose from the urethra to adjacent organs is a relatively uncommon urologic problem that may cause serious complications which arose tardive. Presentation includes a variety of acute or chronic symptoms that depend of the underlying complications. The method of extraction depends on the shape, size and nature of the object and should be tailored according to the condition of the patient. In the present article we present a case of a rubber tube inserted to the urethra for erotic arousal purposes which migrated to the bladder during masturbation.
\end{abstract}

KEY WORDS: Foreign bodies; Urinary tract; Fetishism.

Submitted 29 February 2016; Accepted 25 April 2016

\section{INTRODUCTION}

Insertion of foreign bodies in the urinary tract is a relatively common condition and is due to different aetiologies. It is usually iatrogenic in the upper urinary tract, however in the bladder and urethra it may equally be iatrogenic or due to self-insertion, and rarely due to migration from adjacent organs (1). Self-insertion of foreign bodies in the urethra is an unusual condition and the reasons for it are difficult to comprehend. It is most commonly associated with sexual or erotic arousal of adolescents with psychiatric disorders. Other reasons for urethral manipulation include, drug intoxication, mental confusion, sexual curiosity, sexual arousal and/or a desire to get relief from urinary symptoms. Foreign bodies used for the above mentioned purpose may include flexible or rigid and fragile or strong materials such as needles, bullets and pens, as well as candles, gauzes, etc. Migration from the urethra to adjacent organs is a relatively uncommon urologic problem. The foreign body can remain there for a long time with no or minimal discomfort. In most cases, however, the foreign body causes severe pain, hematuria, and urinary tract infection which rise tardive (2). Treatment approach should also minimize urethral and/or bladder trauma and must ensure the complete extraction of the object. The last must be confirmed by radiology or endoscopy at the end of the extraction procedure. Here, we report an interesting case of a rubber tube inserted to the urethra for erotic arousal purposes which was migrated to the bladder during masturbation and its successful management.

\section{Case report}

A 65-year-old man presented in the emergency department reporting a rubber tube placement in the urethra for sexual pleasure, for more than 36 hours. He reported no dysuria or pain after insertion. Physical examination showed no evidence of foreign body in the urethra. Laboratory findings revealed microscopic hematuria and mild pyuria in routine urine analysis, but the results of the complete blood count and electrolyte profile were normal. $\mathrm{X}$-ray of the pelvis showed a coiled-up radio-opaque shadow in the bladder (Figure 1). Cystoscopy confirmed the presence of a red coloured plastic tube in the bladder lumen (Figure 2). With the use of a crocodile type endoscopic grasper, rearrangement of the tube in order to facilitate its removal due to inconvenient positioning of the tube inside the bladder was successfully done under local anaesthesia and the tube was finally removed. Length of tube was $23 \mathrm{~cm}$ (Figure 3). No urethral and/or bladder trauma occurred. The complete extraction of the object was confirmed by both endoscopy and radiology at the end of the extraction procedure. Postoperative period was uneventful.

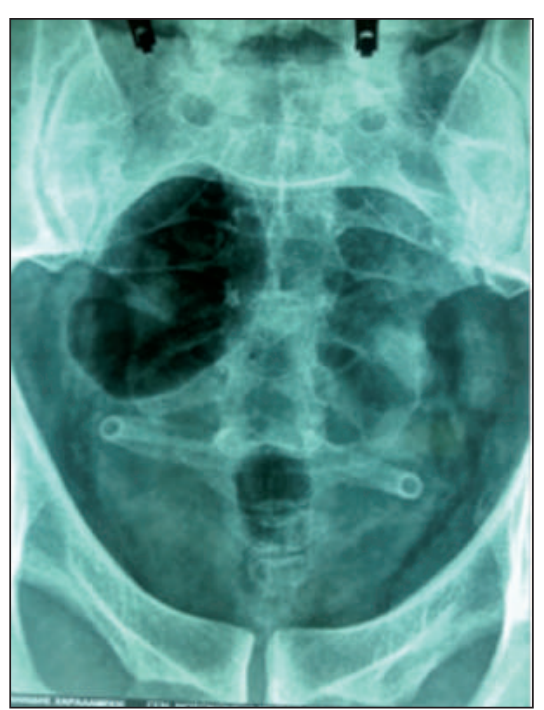

Figure 1.

X-ray of the pelvis shows a coiled-up radio-opaque shadow in the bladder. 


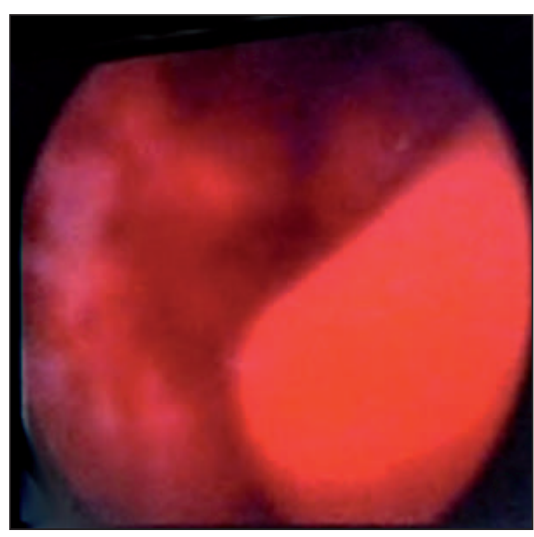

Figure 2.

Cystoscopy image of a red coloured rubber tube in the bladder lumen.

Figure 3.

The length of the red coloured rubber tube is $23 \mathrm{~cm}$.

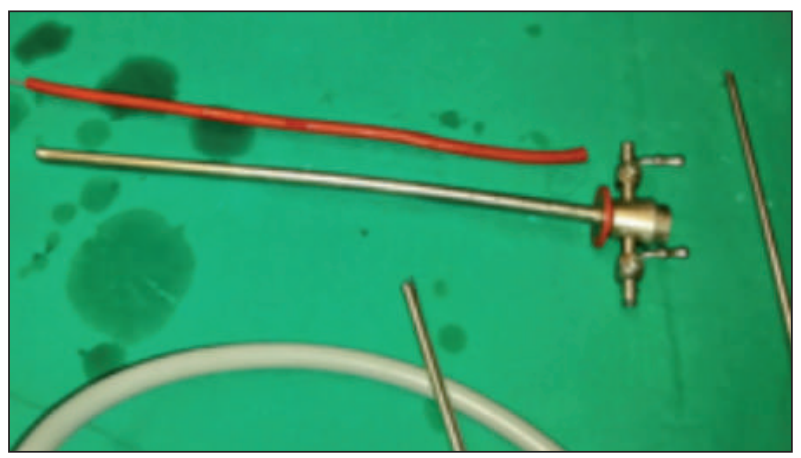

\section{Discussion}

Healthy adult individuals rarely practice insertion of foreign bodies in the urethra. This practice in a sexual context concerns men with urethral manipulation fetishes which are commonly referred with the terms urethral sounding and urethral play. Both can involve the introduction of either soft or rigid items into the meatus of the penis and farther in the urethra and the prostate. Rigid objects such as sounds are usually only inserted about halfway into the glans and can usually be easily retrieved. In contrast, soft objects such as tubes and catheters may be introduced deeper. Some items may even be allowed to curl several times or expand within the bladder. This action in the male may be directly or indirectly associated with stimulation of the prostate gland which produce erotic satisfaction (3). Urethral manipulation may occur both actively or passively associated with fetishism or masochism respectively. Persons who get sexually aroused from the self-insertion of objects into their urethra for autoerotic stimulation purposes falls to the first category and consist the majority of the cases. The second category concerns insertion of foreign bodies in the urethra via medical procedures requested by the person. Although this behaviour shares features with masochism since very few of those who engage in such practices report pain, the association does not seem justified based on the clinical evidence reported (4). In our case, despite careful and discrete history taking it wasn't possible to determine whether the rubber tube was inserted to the urethra by the patient or his sexual companion. According to the literature, a multitude of objects have been found in the urinary bladder. Among others, tubes made of rubber are the most commonly used (5). In contrast to our case, most of the reported cases of foreign body insertion to the urinary tract are characterized by severe symptoms such as pain, hematuria, and urinary tract infection (6). In all cases however, careful history taking, physical examination and imaging evaluation are necessary in order to determine the size and location of the foreign body. This is of outmost importance for the selection of the appropriate method to remove the foreign bodies completely with the minimal damage to the bladder and urethra. Similarly to our case, endoscopic management is reported to be successful however in a few cases open procedures such as suprapubic cystostomy, external urethrotomy or meatotomy are required (7). Our patient refused psychiatric evaluation however it seems that motivation and associated psychosocial issues requires investigation since they are both important in order to prevent future episodes.

\section{ETHICAL APPROVAL}

Written informed consent was obtained from the patient for publication of this case report and its accompanying images. A copy of the written consent is available for review by the Editor-in-Chief of this journal on request.

\section{References}

1. Rieder J, Brusky J, Tran V, et al. Review of intentionally selfinflicted, accidental and iatrogenic foreign objects in the genitourinary tract. Urol Int. 2010; 84:471-475.

2. van Ophoven A, deKernion JB. Clinical management of foreign bodies of the genitourinary tract. J Urol. 2000; 164:274-87.

3. http://en.wikipedia.org/wiki/Urethral_sounding. Accessed on 05/03/2015

4. https://drmarkgriffiths.wordpress.com/tag/urethral-foreign-bodies/. Accessed on 05/03/2015

5. Cho DS, Kim SJ, Choi JB. Foreign bodies in urethra and bladder by implements used during sex behavior. Korean J Urol. 2003; 44:1131-1134.

6. Moon SJ, Kim DH, Chung JH. Unusual foreign bodies in the urinary bladder and urethra due to autoerotism. Int Neurourol J. 2010; 14:186-189.

7. Mitterberger M, Peschel R, Frauscher F, Pinggera GM. Allen key completely in male urethra: a case report. Cases J. 2009; 2:7408. 